\title{
Why Waste a Perfectly Good Abstraction?
}

\author{
Arie Gurfinkel and Marsha Chechik \\ Department of Computer Science, University of Toronto, \\ Toronto, ON M5S 3G4, Canada \\ \{arie, chechik\}@cs.toronto.edu
}

\begin{abstract}
Software model-checking based on the CEGAR framework can be made more precise by separating non-determinism from the lack of information due to abstraction. The two can be modeled individually using four-valued Belnap logic. In addition, this logic allows reasoning about negations effectively and thus enables checking of full CTL. In this paper, we present YASM - a new symbolic software model-checker. Preliminary experience with YASM shows that our implementation can effectively construct and analyze Belnap models without a substantial overhead when compared to its classical counterparts.
\end{abstract}

\section{Introduction}

Symbolic software model-checking, pioneered by the Microsoft's SLAM [1] project, is a technique that works directly on code and checks the program by combining automated predicate abstraction [13] with counterexample-guided abstraction refinement (CEGAR) [6]. The approach is divided into three phases: abstraction, model-checking, and refinement. During the abstraction phase, a theorem-prover is typically used to construct, using a list of predicates, a finite model that approximates the program being verified. The model is analyzed by the model-checker, and counterexamples generated by it are used to find additional predicates, if necessary. The process continues until either the property is successfully proved or disproved, or resources are exhausted.

For example, suppose our goal is to verify whether the line labelled P1 can be reached in the (deterministic) $\mathrm{C}$ program shown in Figure $1 \mathrm{a}$ ). This can be expressed in CTL as $A G(p c \neq \mathrm{P} 1)$. Figure 1 c)-(e) gives a series of predicate programs which are automatically constructed while checking this property. The abstraction in Figure 1(c) is just the control-flow graph of the program, where the symbol ' $*$ ' indicates that the condition was abstracted away, and its value is not known. ' $*$ ' is thus interpreted as "either true or false", and treated as a non-deterministic choice during model-checking. Verifying $A G(p c \neq \mathrm{P} 1)$ on this abstraction yields false. It is possible to resolve nondeterminism so as to reach the line labeled P1, i.e., by exiting the while and entering the if statement. We then check the feasibility of this execution in the concrete program, with the goal of replacing the undesired non-determinism. Specifically, a predicate $x=2$ is needed to determine whether the control flow enters the if statement. The new abstraction is shown in Figure 1d): $x=2$ becomes true during initialization, is not affected by the body of the loop, and is checked in the condition of the if statement. Now, the analysis yields that the property is violated if the loop terminates, and the condition $y \leq 2$ of the loop is added to the list of predicates, yielding an abstraction in Figure1(e). The statement $\mathrm{y}=\mathrm{y}-1$ is abstracted as follows: if $y \leq 2$ is true, then 
behaviour is controlled by the environment, there exists a concrete execution leading to $\mathrm{P} 1$. Thus, we can conclude $A G(p c \neq \mathrm{P} 1)$ fails, without any further refinements or analysis of the feasibility of this execution.

In this paper, we present an approach to proving truth and falsity of reachability properties. It is based on treating unknowns resulting from abstraction, ' $*$ ', differently from unknowns resulting from the environment, non-determinism, as shown in the above example. Our approach is similar to the one taken by Reps and Sagiv [26] in the sense that it uses a logic with additional truth values (we use Belnap logic [4] which is an extension of Kleene logic [22] used in [26]) enabling us to perform both checks during a single analysis phase. The analysis yields one of the following answers: (1) the property holds; (2) the property fails (as in the model in Figure 2(d)); (3) the value of the property depends on the resolution of ' $*$ ', and thus the abstraction needs to be further refined.

We also present an implementation of this approach via a symbolic software modelchecker YASM 1 . Although similar approaches have been studied theoretically, e.g. see [8, 12], we believe this to be the first efficient implementation with performance that is comparable to SLAM and BLAST. The implementation makes use of a number of ideas from existing CEGAR approaches, which we have generalized for our purposes. In particular, our implementation is applicable to programs with non-deterministic controlflow, and is not restricted to reachability analysis.

The rest of this paper is organized as follows. After giving the necessary background in Section 2, we describe, in Section 3, the process of creating and interpreting abstractions of programs we want to check. We discuss three abstract semantics: over-approximation, under-approximation and exact approximation, used in YASM. In Section 4 we describe model-checking of the models constructed via the exact approximation and the use of counterexamples for conclusiveness, generated by the modelchecker, for computing refined abstractions. Exact approximations enable the use of effective techniques for improving the speed and the precision of the analysis. We discuss a few of them in Section 5 We describe the tool and give its performance data in Section 6, and conclude in Section 7 with a comparison of our approach with related work, a summary of the paper, and a discussion of future research directions.

\section{Background}

In this section, we review multi-valued logics, define multi-valued Kripke, and a multivalued version of the modal $\mu$-calculus.

Logics. Boolean logic $\mathbf{2}$ is a set $\{\mathrm{t}, \mathrm{f}\}$ together with the truth ordering relation $\sqsubseteq$, s.t. $\mathrm{f} \sqsubseteq \mathrm{t}$. Conjunction $\wedge$ and disjunction $\vee$ represent meet and join with respect to the truth ordering. Additionally, a negation operator is defined as $\neg t \triangleq f$ and $\neg f \triangleq t$. Kleene logic [22] 3 extends $\mathbf{2}$ with an additional element $\perp$, representing "unknown" information. In this paper, $\perp$ is used to represent ' $*$ ', discussed in Section 1 , The truth ordering of the logic is extended as $f \sqsubseteq \perp$ and $\perp \sqsubseteq \mathrm{t}$, and negation as $\neg \perp=\perp$. We define an additional ordering $\preceq$, that relates values based on the amount of information; thus $\perp \preceq \mathrm{t}$ and $\perp \preceq \mathrm{f}$, so that $\perp$ represents the least amount of information. Belnap logic [4]

\footnotetext{
${ }^{1}$ YASM stands for a Yet Another Software Model-checker.
} 

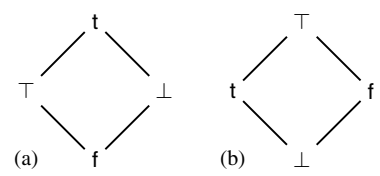

Fig. 3. Belnap logic: (a) truth order; (b) information order

4 extends 3 with an additional element $\top$. The truth ordering is extended so that $\mathrm{f} \sqsubseteq \top$ and $T \sqsubseteq \mathrm{t}$, and negation as $\neg \top=T$, i.e., $T$ is equivalent to $\perp$ with respect to this ordering. Finally, the information ordering is extended by making $\top$ be the largest element, i.e., $\mathrm{f} \preceq \top$ and $\mathrm{t} \preceq \top$. This makes 4 into the smallest structure containing 2 that is a complete distributive lattice under both truth and information orderings. The truth and information orderings of 4 are shown in Figure 3 .

Temporal Logic. Temporal logic properties are specified in propositional $\mu$-calculus $L_{\mu}(A P)$ [23]. Properties are often expressed in CTL, which is a subset of $L_{\mu}$ [7].

Definition 1. Let Var be a set of variable names, and AP be a set of atomic propositions. The logic $L_{\mu}(A P)$ is the set of formulas defined as:

$$
\varphi::=Z|p| \neg \varphi|\varphi \wedge \varphi| \diamond \varphi \mid \mu Z \cdot \varphi(Z)
$$

where $p \in A P, Z \in \operatorname{Var}$, and $\varphi(Z)$ is syntactically monotone in $Z$.

We use the following syntactic abbreviations:

$$
\varphi \vee \psi=\neg(\neg \varphi \wedge \neg \psi) \quad \square \varphi=\neg \diamond \neg \varphi \quad \nu Z \cdot \varphi(Z)=\neg \mu Z \cdot \neg \varphi(\neg Z)
$$

The semantics of $L_{\mu}$ is defined with respect to an $\mathcal{L}$-valued Kripke structures.

Definition 2. An $\mathcal{L}$-valued Kripke structure over a set of atomic propositions $A P$ is a tuple $K=\langle S, \mathcal{L}, R, I\rangle$, where $S$ is a set of states, $\mathcal{L} \in\{\mathbf{2}, \mathbf{3}, \mathbf{4}\}$ is a logic, $R$ : $S \times S \rightarrow \mathcal{L}$ is a transition relation, and $I: A P \rightarrow[S \rightarrow \mathcal{L}]$ is an interpretation of atomic propositions that assigns to each atomic proposition a mapping from states to values in $\mathcal{L}$.

We often refer to $\mathcal{L}$-valued Kripke structures simply as Kripke structures when $\mathcal{L}$ is irrelevant or clear from the context. For a transition relation $R: S \times S \rightarrow \mathcal{L}$, we define the preimage of $Q: S \rightarrow \mathcal{L}$ w.r.t. $R$, pre $[R]:[S \rightarrow \mathcal{L}] \rightarrow[S \rightarrow \mathcal{L}]$, as

$$
\operatorname{pre}[R](Q) \triangleq \lambda s \in S \cdot \bigvee_{t \in S} R(s, t) \wedge Q(t)
$$

pre $[R](Q)$ is a set of states that have an $R$-successor in $Q$. A dual of pre is $w p$ :

$$
w p[R](Q) \triangleq \neg \operatorname{pre}[R](\neg Q)
$$

$w p[R](Q)$ is a set of states whose $R$-successors are all in $Q$.

The semantics of $L_{\mu}$ formula $\varphi$ in a Kripke structure $K$, written $\|\varphi\|_{\sigma}^{K}$, is defined inductively on the structure of the formula, where $\sigma: \operatorname{Var} \rightarrow \mathcal{L}^{S}$ is an object assignment for free variables: 


$$
\begin{array}{ll}
\|p\|_{\sigma}^{K} \triangleq I(p) & \|z\|_{\sigma}^{K} \triangleq \sigma(z) \\
\|\varphi \wedge \psi\|_{\sigma}^{K} \triangleq\|\varphi\|_{\sigma}^{K} \wedge\|\psi\|_{\sigma}^{K} & \|\neg \varphi\|_{\sigma}^{K} \triangleq \neg\|\varphi\|_{\sigma}^{K} \\
\|\mu x \cdot \varphi\|_{\sigma}^{K} \triangleq \operatorname{lfp}^{5}\left(\lambda S \cdot\|\varphi\|_{\sigma[x \mapsto S]}^{K}\right) & \|\nabla \varphi\|_{\sigma}^{K} \triangleq \operatorname{pre}[R]\left(\|\varphi\|_{\sigma}^{K}\right)
\end{array}
$$

where $\operatorname{lfp}^{\sqsubseteq} f$ is the $\sqsubseteq$-least fixpoint of $f$. For a closed $L_{\mu}$ formula $\varphi,\|\varphi\|_{\sigma}^{K}=\|\varphi\|_{\sigma^{\prime}}^{K}$ for any $\sigma$ and $\sigma^{\prime}$, written as $\|\varphi\|^{K}$. For a Kripke structure $K$ and a state $s$, we write $K, s \models \varphi$ to mean $\|\varphi\|^{K}(s)=$ true. Note that when $K$ is 4 -valued, $K, s \not \models \varphi$ does not mean that $K, s \models \neg \varphi$, i.e., proving that $\varphi$ is false is not the same as failing to prove that $\varphi$ is true. Finally, we define $\square L_{\mu}$ and $\diamond L_{\mu}$ to be subsets of $L_{\mu}$, where the modal operations are just $\square$ and $\diamond$, respectively, and negation is allowed only at the level of atomic propositions.

\section{Program Abstraction}

In this section, we show how programs are approximated by Boolean programs and present three approximation semantics.

\subsection{Programs}

Operations. Let $V$ denote the set of program variables. A program is built out of operations $O p s$ of which there are two kinds: (1) an assignment $l:=e$, where $l$ is a variable from $V$ and $e$ is an expression over program variables, and (2) an operation assume $(e)$, where $e$ is a boolean expression. Assume operations are used to model conditional branches. We also use an operation skip as a syntactic abbreviation for assume (t).

Programs as Control Flow Graphs. A Control Flow Graph CFG is a structure $G=$ $\langle L o c, \delta\rangle$, where $L o c$ is a finite set of locations, and $\delta: L o c \times L o c \rightarrow \mathbf{2}$ is a transition relation. A program is modeled by a labeled $\mathrm{CFG}\langle G, \tau\rangle$, where $\tau$ labels each edge of the CFG $G$ with an operation from Ops. A CFG corresponding to the program in Figure 1 a) is shown in Figure1(b).

Programs as Kripke Structures. A state is a type-correct valuation of all program variables. We use $S$ to denote the set of all states, and $s(x)$ to denote the value of the variable $x$ in $s$. Each operation op corresponds to a transition relation $\mathrm{S}(o p)$ defined as:

$$
\mathrm{S}(o p)(s, t) \Leftrightarrow t= \begin{cases}s & \text { if op is assume }(e) \text { and } s \models e \\ s[l \mapsto s . e] & \text { if op is } l:=e\end{cases}
$$

Finally, a program $\operatorname{Prg}=\langle G, \tau\rangle$ corresponds to a Kripke structure $K_{\operatorname{Prg}} \triangleq\langle L o c \times$ $\left.S, \mathbf{2}, R_{P r g}, I_{P r g}\right\rangle$, where $R$ and $I$ are defined as:

$$
\begin{aligned}
& R_{\operatorname{Prg}}(\langle l, s\rangle,\langle k, t\rangle) \triangleq \delta(l, k) \wedge(\mathrm{S}(\tau(l, k)))(s, t) \\
& I_{\operatorname{Prg}}(p c=j)(\langle l, s\rangle) \triangleq(l=j) \\
& I_{P r g}(e)(\langle l, s\rangle) \quad \triangleq s \models e
\end{aligned}
$$

and $e$ is a boolean expression.

The semantics of $L_{\mu}$ is extended to programs in the obvious way: a program Prg satisfies $\varphi$ iff the corresponding Kripke structure $K_{P r g}$ satisfies $\varphi$. 


\subsection{Boolean Programs}

Boolean Operations. Let $P=\left\{p_{1}, \ldots, p_{n}\right\}$ be a set of quantifier-free first-order boolean predicates over program variables $V$. A Boolean (or Predicate) program [2] is a program constructed out of Boolean operations BOps. As before, the operations are divided into two kinds: (1) a parallel assignment $p_{1}:=e_{1}, \ldots, p_{n}:=e_{n}$, and (2) an operation assume $(e)$. We refer to elements of a parallel assignment as updates, e.g., $p_{1}:=e_{1}, p_{2}:=e_{2}$ consists of two updates, for predicates $p_{1}$ and $p_{2}$, respectively. The expressions on the right-hand-side of the assignment and in the argument of the assume operation are partial boolean expressions with the following grammar:

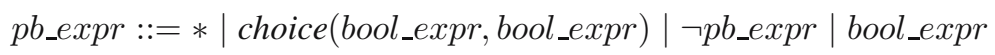

Intuitively, $*$ stands for an unknown expression, and choice $(a, b)$ - for an expression that evaluates to true when $a$ is true, to false when $b$ is true, and whose value is unknown otherwise. In Boolean programs, we use skip as a syntactic abbreviation for a parallel assignment $p_{1}:=\operatorname{choice}\left(p_{1}, \neg p_{1}\right), \ldots, p_{n}:=\operatorname{choice}\left(p_{n}, \neg p_{n}\right)$, and $\neg \operatorname{choice}(a, b)$ for choice $(b, a)$. As before, a Boolean program is a CFG whose edges are labeled with operations from BOps.

Syntactic Abstraction. We now show how a Boolean program BPrg is used to approximate a program $\operatorname{Prg}$ by describing the behavior of $\operatorname{Prg}$ using a finite set of predicates. We present the approximation in a bottom-up fashion, starting with approximation of expressions, and ending with approximation of programs.

A partial boolean expression pe approximates a boolean expression $e$ (denoted as $p e \preceq e$ ), if (a) $p e$ is a boolean expression logically equivalent to $e$, (b) $p e$ is the * expression, (c) pe is of the form choice $(a, b)$ and $a$ logically implies $e$, and $b$ logically implies $\neg e$. For example, $y>0$ is approximated by choice $(y>1, f)$. Note that from the perspective of the approximation, $*$ is equivalent to choice $(f, f)$.

The approximation is extended to the assume operations in the obvious way: assume $(p e) \preceq \operatorname{assume}(e)$ iff $p e \preceq e$, e.g., assume $(y>0)$ is approximated by assume $($ choice $(y>1, \mathrm{f}))$. A single update $p:=\operatorname{choice}(a, b)$ approximates an assignment $l:=e$ if $\operatorname{choice}(a, b)$ approximates the weakest pre-condition of the predicate $p$ with respect to the assignment. In other words, $a$ approximates the condition under which $p$ becomes true after the assignment, and $b$ approximates the condition under which $p$ becomes false. For example, a program assignment $y:=y-1$ is approximated by $(y \leq 2):=\operatorname{choice}(y \leq 2, \mathrm{f})$. Finally, a parallel assignment $A$ approximates an assignment $l:=e$ if all update operations of $A$ approximate $l:=e$. For example, $y:=y-1$ is approximated by $(y \leq 2):=\operatorname{choice}(y \leq 2, \mathrm{f}),(x=2):=\operatorname{choice}((x=2), \neg(x=2))$.

We say that a Boolean program $B P r g=\left\langle G, \tau_{B}\right\rangle$ approximates a program Prg $=$ $\langle G, \tau\rangle$ if each operation of BPrg approximates the corresponding operation of Prg. Since we have not yet given an operational semantics to Boolean programs, we call this approximation a syntactic predicate abstraction. There are standard techniques to compute such abstractions [1, 13].

\subsection{Three Semantics of Boolean Programs}

In order to evaluate temporal properties on Boolean programs, we must equip them with Kripke semantics. The only difficulty is to find a proper way to model the partial expres- 
sions, i.e., $*$ and choice $(a, b)$. In this section, we describe three choices for this approximation: (a) an over-approximating semantics where "unknown" is modeled as a nondeterministic choice between true and false - this is the semantics used by most existing model-checkers such as SLAM [2] and BLAST [20]; (b) an under-approximating semantics where "unknown" is modeled by a partial assignment; and (c) the exact semantics that uses Belnap logic to combine over- and under-approximation - this is the semantics used by our model-checker YASM. The three semantics are illustrated on a parallel assignment $A:(y \leq 2):=\operatorname{choice}(y \leq 2, \mathrm{f}),(x=2):=\operatorname{choice}((x=2), \neg(x=2))$ that approximates $y:=y-1$ using predicates $y \leq 2$ and $x=2$.

Over-Approximation. In this case, a state is a boolean valuation of predicates, i.e., it is an element of $\mathbf{2}^{P}$. Each operation bop in $B O p$ is associated with a transition relation $\mathrm{O}($ bop $) \subseteq \mathbf{2}^{P} \times \mathbf{2}^{P}$, such that abstract states $a$ and $b$ are not connected if we can conclude from the boolean operation that there is no transition between the corresponding states of the concrete program. That is, if the current state $a$ does not satisfy the precondition for $p$ to become false, $a$ has a successor, $b$, in which $p$ is true.

Formally, the semantics of an update operation is

$$
\mathrm{O}(p:=\operatorname{choice}(q, r))(a, b(p)) \Leftrightarrow(b(p)=\mathrm{t} \text { and } a \not \models r) \text { or }(b(p)=\mathrm{f} \text { and } a \not \models q)
$$

and semantics of a parallel assignment is the conjunction of all of its updates:

$$
\mathrm{O}\left(\left\{p_{i}:=\operatorname{choice}\left(q_{i}, r_{i}\right)\right\}_{i=1}^{n}\right)(a, b) \Leftrightarrow \bigwedge_{i=1}^{n}\left(\mathrm{O}\left(p_{i}:=\operatorname{choice}\left(q_{i}, r_{i}\right)\right)\left(a, b\left(p_{i}\right)\right)\right)
$$

For our running example, a part of a transition relation $\mathrm{O}(A)$ is shown in Figure 4 (a). Note that a state $a_{1}$ corresponding to concrete states where $(y \not \leq 2)$ and $x=2$ has two outgoing transitions, to states $a_{0}$ and $a_{1}$, indicating that it is possible for $y \leq 2$ to nondeterministically become true or false in the next state. That is, the fact that the value of $y \leq 2$ is unknown in the next state is modeled by non-determinism.

Finally, the semantics of the assume operator is:

$$
\mathrm{O}(\text { assume }(e))(a, b) \Leftrightarrow a \not \models \neg e \text { and } \mathrm{O}(\text { skip })(a, b)
$$

Under-Approximation. In this case, a state is a partial valuation of predicates, i.e, an element of $\mathbf{3}^{P}$, or a "tri-vector" [1]. Each operation bop $\in B O p$ is associated with a transition relation $\mathrm{U}(b o p) \subseteq 3^{P} \times 3^{P}$, such that each predicate $p$ is true in the next state, $b$, only if the current state, $a$, satisfies a precondition for $p$ to become true.

Formally, the semantics of an update operation is

$$
\mathrm{U}(p:=\operatorname{choice}(q, r))(a, b(p)) \Leftrightarrow(b(p)=\mathrm{t} \text { and } a \models q) \text { or }(b(p)=\mathrm{f} \text { and } a \models r) \text { or }(b(p)=\perp)
$$

and semantics of a parallel assignment is the conjunction of all of its updates:

$$
\mathrm{U}\left(\left\{p_{i}:=\operatorname{choice}\left(q_{i}, r_{i}\right)\right\}_{i=1}^{n}\right)(a, b) \Leftrightarrow \bigwedge_{i=1}^{n}\left(\mathrm{U}\left(p_{i}:=\operatorname{choice}\left(q_{i}, r_{i}\right)\right)\left(a, b\left(p_{i}\right)\right)\right)
$$

For our running example, a part of a transition relation $\mathrm{U}(A)$ is shown in Figure 4 (b). Here, state $a_{1}$ has a single outgoing transition to state $a_{3}$ indicating that in the next state the value of $y \leq 2$ is unknown, and $x=2$ remains true. 


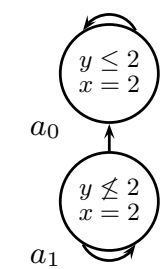

(a)

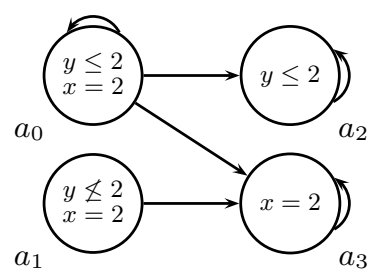

(b)

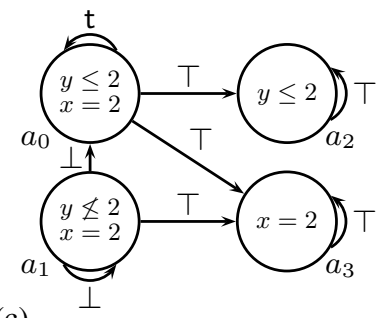

(c)

Fig. 4. Fragment of a transition relation: (a) over-approximation, (b) under-approximation, and (c) exact approximation

Finally, the semantics of the assume operator is:

$$
\mathrm{U}(\text { assume }(e))(a, b) \Leftrightarrow a \models e \text { and } \mathrm{U}(\text { skip })(a, b)
$$

Exact Approximation. This approximation combines the over- and underapproximating semantics in a single $\mathbf{4}$-valued model. Thus, the states are partial valuations of predicates, i.e., elements of $3^{P}$. Each operation bop in $B O p$ is associated with a 4 -valued transition relation $\mathrm{E}(b o p): 3^{P} \times 3^{P} \rightarrow 4$. Intuitively, a transition is $\mathrm{t}$ if it appears both in the over- and the under-approximations, $\perp$ if it appears only in the overapproximation, and $T$ if it appears only in the under-approximation. For our running example, a part of a transition relation $\mathrm{E}(A)$ is shown in Figure 4 (c).

To define the semantics formally, we first introduce a function $\operatorname{eval}()$ :

$$
\operatorname{eval}(\varphi, a) \triangleq \begin{cases}\mathrm{t} & \text { if } a \models \varphi \\ \mathrm{f} & \text { if } a \models \neg \varphi \\ \perp & \text { if } a \not \models \varphi \text { and } a \not \models \neg \varphi\end{cases}
$$

Then, the semantics of an update is

$$
\mathrm{E}(p:=\operatorname{choice}(q, r))(a, b(p))= \begin{cases}\operatorname{eval}(\operatorname{choice}(q, r), a) & \text { if } b(p)=\mathrm{t} \\ \operatorname{eval}(\neg \operatorname{choice}(q, r), a) & \text { if } b(p)=\mathrm{f} \\ \top & \text { if } b(p)=\perp\end{cases}
$$

and the semantics of a parallel assignment is the conjunction of all of its updates:

$$
\mathrm{E}\left(\left\{p_{i}:=\operatorname{choice}\left(q_{i}, r_{i}\right)\right\}_{i=1}^{n}\right)(a, b) \Leftrightarrow \bigwedge_{i=1}^{n}\left(\mathrm{E}\left(p_{i}:=\operatorname{choice}\left(q_{i}, r_{i}\right)\right)\left(a, b\left(p_{i}\right)\right)\right)
$$

Finally, the semantics of the assume operation is:

$$
\mathrm{E}(\operatorname{assume}(e))(a, b) \triangleq \operatorname{eval}(e, a) \wedge \mathrm{E}(\operatorname{skip})(a, b)
$$

The semantics of operations is extended to Boolean programs in an obvious way. Thus, each semantics associates a Boolean program with a Kripke structure. For example, the Kripke structure corresponding to the boolean program in Figure 2(d) is shown in Figure 5(a). The following theorem shows that over- and under-approximating semantics preserve universal and existential fragments of $L_{\mu}$, respectively, whereas the exact semantics preserves full $L_{\mu}$. 

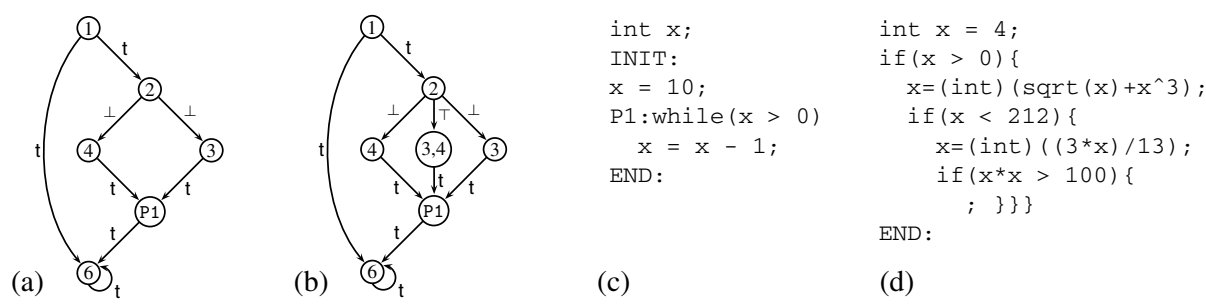

(d)

Fig. 5. (a) a Kripke structure corresponding to the Boolean program in Figure2 2 d); (b) improving precision of if; (c) and (d) two example programs

Theorem 1. Let Prg and BPrg be a program and its Boolean abstraction, respectively. Then, for an abstract state, a, and a corresponding concrete state, $s$, the following holds:

$$
\begin{array}{lll}
\text { 1. } \forall \varphi \in \square L_{\mu} \cdot \mathrm{O}(B \operatorname{Prg}), a \models \varphi & \Rightarrow & \operatorname{Prg}, s=\varphi \\
\text { 2. } \forall \varphi \in \diamond L_{\mu} \cdot \mathrm{U}(B \operatorname{Prg}), a \models \varphi & \Rightarrow & \operatorname{Prg}, s=\varphi \\
\text { 3. } \forall \varphi \in L_{\mu} \cdot \mathrm{E}(B \operatorname{Prg}), a \models \varphi & \Rightarrow \quad \operatorname{Prg}, s=\varphi
\end{array}
$$

The over-approximating semantics has been used in standard software model-checking tools to prove truth of $A G$ properties. Our approach uses the exact semantics, which enables us to prove truth and falsity of such properties. We discuss it in the next section.

\section{Abstract Model-Checking}

To model-check a temporal logic formula $\varphi$ in a state $s$ and location $l$ of a Boolean program $B P r g$, we use the techniques of Section 3 to construct a 4-valued Kripke structure $K_{B P r g}$ and then compute the value of $\|\varphi\|^{K_{B P r g}}(\langle l, s\rangle)$. The latter step involves multivalued model-checking, e.g., using the algorithm implemented in $\chi$ Chek [5]. In Section 4.1 we describe how to perform model-checking with Belnap logic using standard BDD packages.In Section 4.2, we show how to find additional predicates to refine the abstraction if the result of model-checking a formula $\varphi$ is inconclusive.

\subsection{Model-Checking}

A symbolic multi-valued model-checking algorithm depends on an efficient representation and manipulation of Belnap functions, i.e., functions from some set $S$ into 4 . These functions are represented in YASM as BDDs using the ideas below. First, any set $S$ can be encoded by $r$ boolean variables $v_{1}, \ldots, v_{r}$, for a sufficiently large $r$. Thus, we only need to find a representation for Belnap functions whose domain is $\mathbf{2}^{r}$. Second, any Belnap function $f: \mathbf{2}^{r} \rightarrow \mathbf{4}$ can be represented by a pair of boolean functions $\left\langle f_{\top}, f_{\perp}\right\rangle$ over $\mathbf{2}^{r}$ [16], where $f_{\top}$ is $\lambda x \cdot f(x) \sqsupseteq \top$ and $f_{\perp}$ is $\lambda x \cdot f(x) \sqsupseteq \perp$. With this decomposition, $f(x)$ is equivalent to $\left(f_{\top}(x) \wedge \top\right) \vee\left(f_{\perp}(x) \wedge \perp\right)$. The following equivalences enable the direct computation of conjunction and disjunction of Belnap functions: (a) $f \wedge g=\left\langle f_{\top} \wedge g_{\top}, f_{\perp} \wedge g_{\perp}\right\rangle$; (b) $f \vee g=\left\langle f_{\top} \vee g_{\top}, f_{\perp} \vee g_{\perp}\right\rangle$; (c) $\neg f=\neg\left\langle f_{\top}, f_{\perp}\right\rangle=\left\langle\neg f_{\perp}, \neg f_{\top}\right\rangle$. Thus, we can represent each Belnap function by a pair of BDDs, one for each boolean function in the decomposition. Third, a pair of boolean 
functions $\langle f, g\rangle$ over variables $v_{1}, \ldots, v_{r}$ is represented by a single boolean function $h$ with a new boolean variable $z$, using the encoding $h=z \wedge f \vee \neg z \wedge g$. So, Belnap functions can be represented and manipulated as standard BDDs at the expense of one additional variable. Furthermore, as many other symbolic model-checkers, we use the control-flow graph to partition the transition relation and its pre-image computation.

\subsection{Abstraction Refinement}

Whenever model-checking $\varphi$ is inconclusive, our model-checker produces a behaviour of the system explaining why this is the case [15,14]. So, we can use any of the existing techniques, e.g., [21], to determine whether this trace is feasible and use it to obtain additional predicates to refine the abstraction. However, in the multi-valued framework, checking feasibility of the trace is not necessary: we know exactly which part is inconclusive, and concentrate the refinement on it.

For example, consider the program in Figure 2(c), its abstraction in Figure2(d), and the corresponding Kripke structure in Figure 5 a). Since the abstraction is built without any predicates, the Kripke structure is essentially equivalent to the CFG of the program. In this abstraction, $\|E F(p c=\mathrm{P} 1)\|(1)$ is inconclusive, i.e., $\perp$, which is exemplified by a path $1,2,4, \mathrm{P} 1$ with an $\perp$-transition between states 2 and 4 . In this example, the $\perp$-transition is the result of executing a boolean operation assume $(*)$ that syntactically abstracts assume $(x>0)$ in the concrete program. Thus, the value of the predicate $x>0$ is required to make the proof conclusive, which is done by refining the abstraction with this predicate, and repeating model-checking on the refined program.

In general, a path exemplifying why the result of model-checking is inconclusive always contains at least one $\perp$-transition. Suppose such a transition is between states $\langle k, a\rangle$ and $\langle l, b\rangle$, where $k$ and $l$ are program locations, and $a$ and $b$ are boolean valuations of predicates. By construction, this transition corresponds to a boolean operation bop such that $\mathrm{E}(b o p)(a, b)=\perp$. If bop is a parallel assignment, then by the definition of the exact semantics there exists a predicate $p$ and an update of the form $p:=\operatorname{choice}(q, r)$ in bop such that $a \not \models q$ and $a \not \models r$. The idea is to refine the update, by strengthening the expressions $q$ and $r$ by the precondition for $p$ to become true after execution of the concrete operation op corresponding to bop. This is done by refining the Boolean program with the predicate corresponding to the weakest precondition of $p$ with respect to $o p$, i.e., $w p[o p](p)$.

For example, suppose we have model-checked a Boolean program with two predicates $y \leq 2$ and $x=2$, and the cause of inconclusiveness is a $\perp$-transition between states $a=\{(y \leq 2) \mapsto \mathrm{f},(x=2) \mapsto \mathrm{t}\}$ and $b=\{(y \leq 2) \mapsto \mathrm{t},(x=2) \mapsto \mathrm{t}\}$ Further, assume that the corresponding boolean operation is $(y \leq 2):=\operatorname{choice}(y \leq 2, \mathrm{f}),(x=$ $2):=$ choice $(x=2, x \neq 2)$, which is the result of abstracting the concrete operation $y:=y-1$. The transition is unknown since the prestate $a$ does not guarantee that $y \leq 2$ is true or false in the next state, i.e., $a \not \forall y \leq 2$ and $a \not \forall \mathrm{f}$. We can then refine the Boolean program by adding the predicate $w p[y:=y-1](y \leq 2)=(y \leq 3)$.

The above approach to abstraction-refinement is not limited to reachability properties. Our multi-valued model-checker can provide explanations to inconclusiveness of arbitrary CTL properties in the form of proofs [15], which we mine for additional predicates. 


\section{Exploiting Exact Approximations}

The use of precise (Belnap) abstractions, described in Section 3, opens way to creating a number of techniques for improving the speed and the precision of the analysis. In this section, we discuss two of them.

Reusing Results of Previous Abstractions. One of the obvious limitations of the CEGAR framework is the fact that intermediate results are not shared between successive abstraction-refinement iterations. For example, consider applying the framework to check whether the property $E F(p c=\mathrm{END})$ holds in location INIT in the program in Figure 5](c).

In the first iteration, we conclude that reachability of END depends on the value of $x>0$, which is added to the list of predicates. During the model-checking phase of the second iteration, it is proved that END is reachable from $\mathrm{P} 1$ if $x \leq 0$, i.e., $\| E F(p c=$ END $) \|(\langle P 1,\{(x>0) \mapsto f\}\rangle)$ holds in the corresponding Kripke structure. Additionally, during the refinement phase, a new predicate $x>1$ is added. The third iteration once again reproves that END is reachable from $\mathrm{P} 1$ provided that $x \leq 0$ or $x \leq 1$, adding a predicate $x>2$. The process continues, repeating the work done at the previous iterations, until all predicates of the form $x>i$, where $0 \leq i \leq 10$, are added, and termination of the loop is established.

To reuse results of previous computations, we must first identify which results are preserved between iterations. For a program $\operatorname{Prg}$, let $K_{P}$ be a Kripke structure abstracting $\operatorname{Prg}$ using predicates $P=\left\{p_{1}, \ldots, p_{n}\right\}$, constructed during an iteration $i$, and let $K_{P^{\prime}}$ be a Kripke structure constructed during the $i+1$ iteration using predicates $P^{\prime}=P \cup\left\{p_{n+1}\right\}$. From the construction of the abstractions, $\|\varphi\|^{K_{P}}(\langle l, u\rangle) \preceq$ $\|\varphi\|^{K_{P^{\prime}}}(\langle l, v\rangle)$ for a formula $\varphi$ and those states $\langle l, v\rangle$ of $K_{P}$ and $\langle l, u\rangle$ of $K_{P^{\prime}}$, where $v\left(p_{i}\right)=u\left(p_{i}\right)$ for all $i \leq n$ (i.e., $v$ and $u$ agree on the values of the first $n$ predicates). In particular, if the concretization of $v$ is not empty, then, if $\varphi$ is either $\mathrm{t}$ or $\mathrm{f}$ in $\langle l, u\rangle$, it is correspondingly $\mathrm{t}$ or $\mathrm{f}$ in $\langle l, v\rangle$. This allows us to use $\|\varphi\|^{K_{P}}$, the result of model-checking $\varphi$ on $K_{P}$, to help compute $\|\varphi\|^{K_{P^{\prime}}}$ Formally, we define $D_{P}$ as follows:

$$
D_{P}\left(\left\langle l,\left\langle u_{1}, \ldots, u_{n+1}\right\rangle\right\rangle\right)= \begin{cases}\mathrm{t} & \text { if }\|\varphi\|^{K_{P}}\left(\left\langle l,\left\langle u_{1}, \ldots, u_{n}\right\rangle\right\rangle\right)=\mathrm{t} \\ \mathrm{f} & \text { otherwise }\end{cases}
$$

The function $D_{P}$ is an under-approximation of $\|\varphi\|^{K_{P^{\prime}}}$ : for any state $s$ of $K_{P^{\prime}}$ with a non-empty concretization, $D_{P}(s) \sqsubseteq\|\varphi\|^{K_{P^{\prime}}}(s)$. If $\varphi$ is computed using a least fixpoint, e.g., $\varphi=E F \psi, D_{P}$ can be used as the starting point in computing $\varphi$ on $K_{P^{\prime}}$. For formulas that are computed using a greatest fixpoint, an over-approximation with respect to the truth ordering can be constructed and used in a similar manner. For a formula $\varphi=E F \psi$, the above optimization computes, at iteration $i+1$, reachability of states proved to satisfy $E F \psi$ at iteration $i$. In our example, this results in $E F(p c=$ END) during the first and the second iteration, $E F(p c=\operatorname{END} \vee(p c=\mathrm{P} 1 \wedge x \leq 0))$ during the third, $E F(p c=\operatorname{END} \vee(p c=\mathrm{P} 1 \wedge x \leq 1))$ during the fourth, etc. In the standard approach, we would have been checking $E F(p c=$ END) from scratch after each refinement. 
Handling Conditional Statements. In this paper, every abstract state corresponds to a unique control flow location, which simplifies construction of the abstract model but limits its precision. Recall that the goal of checking our program in Figure 2(d) and its corresponding Kripke structure in Figure 5 (a) was to establish whether the location $p c=\mathrm{P} 1$ is reachable. Intuitively, model-checking begins by labeling node $\mathrm{P} 1$ by t, i.e., $\mathrm{P} 1$ is reachable from itself, and then propagates this labeling along the edges of the Kripke structure. Thus, in the second iteration, nodes $p c=4$ and $p c=3$ are labeled by t, i.e., $p c=\mathrm{P} 1$ is definitely reachable from these nodes. In the third iteration, we run into a problem. We would like to conclude that $p c=\mathrm{P} 1$ is reachable from the node $p c=2-$ it is reachable from both branches of the if-statement. However, according to our algorithm, the value at $p c=2$ is obtained by (a) propagating the labeling of $p c=3$ through the $(2,3)$-edge, (b) propagating the labeling of $p c=4$ through the $(2,4)$-edge, and (c) taking a disjunction of (a) and (b). Thus, after the third iteration, the node $p c=2$ is labeled with $(\perp \wedge \mathrm{t}) \vee(\perp \wedge \mathrm{t})=\perp$, allowing us to conclude only that $p c=\mathrm{P} 1$ is $\perp$-reachable from $p c=2$.

The cause of this problem is that in our abstract domain, we cannot express that one of the branches of the if-statements is taken, although we do not know which. One solution is to increase the abstract domain to include a state corresponding to several control flow locations. Figure 5 (b) shows a possible abstraction with an additional abstract state $p c=(3,4)$, corresponding to the set of program states in which the control location is either 3 or 4 . It has a t-transition to $p c=\mathrm{P} 1$ since all states corresponding to it have a transition there, and has a $T$-transition from $p c=2$, indicating that the execution of the if-statement definitely results in the control passing to either location 3 or 4 . In this case, after the second iteration of the model-checking algorithm, nodes $\mathrm{P} 1,3,4$, and $(3,4)$ are labeled with $\mathrm{t}$, and the third iteration results in the desired result: $(\perp \wedge \mathrm{t}) \vee(\top \wedge \mathrm{t}) \vee(\perp \wedge \mathrm{t})=\mathrm{t}$.

Additional abstract states solve our problem; however, they can significantly increase the size of the abstract model and complicate the abstraction process. In YASM, we take a different approach, similar in spirit to "hyper-transitions" (e.g., [24, 27, 9]). Instead of increasing the abstract domain, we use the fact that for any concrete (2valued) left-total transition relation $R, w p[R](Q) \subseteq$ pre $[R](Q)$ - if all successors of a state $s$ are in $Q$, then at least one successor is in $Q$. Thus, the pre-image computation of an abstract transition relation $R_{a}$ can be augmented from $\operatorname{pre}\left[R_{a}\right](Q)$ to pre $\left[R_{a}\right](Q) \vee\left(w p\left[R_{a}\right](Q) \succeq \mathrm{t}\right)$, i.e., a state is assigned $\mathrm{t}$ if either it has a definite successor in $Q$, or all of its non-f successors are definitely in $Q$. In our example, this changes the third iteration of model-checking of the Kripke structure in Figure 5 a) as follows: in addition to computing pre along the edges $(2,3)$ and $(2,4), w p$ along each edge is computed to be $\neg \perp \vee \mathrm{t}=\mathrm{t}$, and the node $p c=2$ is marked with $\mathrm{t}$ as desired. Thus, we can obtain a conclusive abstraction without the need to add the predicate $x>0$.

Our approach also enables us to give definite results for certain programs with nonlinear predicates. Consider the program in Figure 5(d). If the goal of a successful model-checking run is to examplify the path to END (as is the case in standard software model-checking approaches), the presence of complex mathematical operations will make the theorem-proving quite difficult, if not impossible. Our approach enables us to avoid these problems: we simply conclude that the path to END exists, whether 
it goes through the nested if statement or not. For such cases, we effectively perform context-sensitive slicing, removing parts of the abstraction which are not necessary to achieve a conclusive answer.

\section{Experiments}

The techniques described in this paper have been implemented in a software modelchecker YASM. YASM is written in JAVA and uses theorem prover CVC Lite [3] to approximate program statements, and CUDD [28] library as a decision diagram engine.

Table 1 summarizes the performance of YASM on several programs based on the examples distributed with BLAST [20]. The experiments were performed on a Pentium $42.4 \mathrm{GHz}$ machine running Linux 2.4.20. For each experiment, we list the number of iterations required by the abstraction-refinement phase, the final number of predicates, the overall model-checking time, and the final analysis result. For example, running YASM on a 4065-line qpmouse program took three iterations and yielded two predicates, in 2.5 seconds, whereas BLAST solved this problem in 1 second, also using two predicates. For every example, we checked whether an error condition is unreachable, which holds everywhere except gpmouse_err.

Table 1. Experimental results

\begin{tabular}{|l|r|r|r|r|r|r|r|}
\hline Name & \multirow{2}{*}{ LOC } & \multicolumn{3}{|c|}{ YASM } & Result & \multicolumn{2}{|c|}{ BLAST } \\
\cline { 3 - 5 } & & Iterations & \# of Pred & Time (sec) & & Time (sec) \# of Pred \\
\hline \hline tlan & 6885 & 4 & 3 & 15.4 & $\mathrm{t}$ & 52 & 9 \\
qpmouse & 4065 & 3 & 2 & 2.5 & $\mathrm{t}$ & 1 & 2 \\
qpmouse_err & 4065 & 12 & 20 & 5.7 & $\mathrm{f}$ & - & - \\
s3_srvr & 2261 & 3 & 30 & 2.9 & $\mathrm{t}$ & 16.6 & 15 \\
s3_srvr.3 & 2240 & 3 & 38 & 25.1 & $\mathrm{t}$ & 152 & 20 \\
\hline
\end{tabular}

For these experiments, YASM was configured to prefer adding new predicates instead of computing a more precise abstraction. Our results clearly show that the running time of YASM is comparable to that of BLAST. We ran the latter as a baseline, to determine a reasonable performance for a software model-checker: a more direct comparison is not possible because the techniques used in the two model-checkers are significantly different. We do not report the results of running BLAST on qpmouse_err because the answer it gives when error is reachable is unsound: the paths reported by the tool are often infeasible.

\section{Conclusion and Related Work}

In this paper, we have presented YASM - a BDD-based software model-checker that combines automatic predicate abstraction-refinement with reasoning over Belnap logic. Our experience indicates that the CEGAR framework can be successfully extended to do proofs of reachability and proofs of unreachability, using the same abstraction. This 
approach allows us to shorten the abstraction-refinement cycle, is applicable to programs with non-deterministic control-flow, and provides support for model-checking of arbitrary CTL formulas.

There has been a lot of progress in applying automatic predicate abstraction of Graf and Saïdi [13] to software model-checking. The approach closest to ours is the one taken by SLAM [1] - YASM simply reinterprets SLAM's boolean programs using 4valued semantics. Like our work, [25] makes a distinction between "non-deterministic" and "unknown" transitions and shows that performance of an explicit-state software model-checker is improved by guiding it towards the former. In our terminology, this would mean guiding the search to prefer non- $\perp$ transition, which happens automatically in our (symbolic) approach.

4-valued Kripke structures and their application to abstraction are equivalent to Mixed Transition Systems [8, 18]. They can also be seen as an extension of Modal Transition Systems [11] that are defined using Kleene logic.

We are not the first to use multi-valued logic to model abstraction in model-checking. Specifically, Kleene logic has been previously applied to reason about abstractions [26], and suggested as a basis for abstract model-checking [11]. Belnap logic has also been used to model abstraction in the context of (G)STEs [19] in a manner similar to ours.

Models based on Kleene logic have been used in [10] to separate handling of "unknown" and "non-determinism". Unlike our work or that of [8], it does not account for the relationship between abstract states, i.e., the case where $\gamma(a) \subseteq \gamma(b)$ for some abstract states $a$ and $b$. It uses a (rather expensive [10, 17]) generalized model-checking approach and does not address the issue of generating counterexamples which are essential for an application of the CEGAR framework.

We are currently working on an implementation of YASM that combines exact approximations with function summaries for handling recursive functions. This is subject of a forthcoming paper.

\section{Acknowledgments}

We are grateful to Xin Ma, Kelvin $\mathrm{Ku}$ and Shiva Nejati for their help implementing, evaluating and improving YASM, and to Ou Wei for thoroughly reading an earlier draft of this paper and for many interesting discussions. We would like to acknowledge the financial support provided by NSERC. The first author has also been partially supported by an IBM Ph.D. Fellowship.

\section{References}

1. T. Ball, A. Podelski, and S. Rajamani. "Boolean and Cartesian Abstraction for Model Checking C Programs". STTT, 5(1):49-58, 2003.

2. T. Ball and S. Rajamani. "The SLAM Toolkit". In Proceedings of CAV'01, volume 2102 of LNCS, pages 260-264, 2001.

3. C. Barrett and S. Berezin. "CVC Lite: A New Implementation of the Cooperating Validity Checker". In Proceedings of CAV'04, volume 3114 of LNCS, pages 515-518, 2004.

4. N.D. Belnap. "A Useful Four-Valued Logic". In Dunn and Epstein, editors, Modern Uses of Multiple-Valued Logic, pages 30-56. Reidel, 1977. 
5. M. Chechik, B. Devereux, and A. Gurfinkel. " $\chi$ Chek: A Multi-Valued Model-Checker”. In Proceedings of CAV'02, volume 2404 of LNCS, pages 505-509, 2002.

6. E. Clarke, O. Grumberg, S. Jha, Y. Lu, and H. Veith. "Counterexample-Guided Abstraction Refinement for Symbolic Model Checking". Journal of the ACM, 50(5):752-794, 2003.

7. E. Clarke, O. Grumberg, and D. Peled. Model Checking. MIT Press, 1999.

8. D. Dams, R. Gerth, and O. Grumberg. "Abstract Interpretation of Reactive Systems". ACM TOPLAS, 2(19):253-291, 1997.

9. L. de Alfaro, P. Godefroid, and R. Jagadeesan. "Three-Valued Abstractions of Games: Uncertainty, but with Precision”. In Proceedings of LICS'04, pages 170-179, 2004.

10. P. Godefroid. "Reasoning about Abstract Open Systems with Generalized Module Checking”. In Proceedings of EMSOFT'2003, volume 2855 of LNCS, pages 223-240, 2003.

11. P. Godefroid, M. Huth, and R. Jagadeesan. "Abstraction-based Model Checking using Modal Transition Systems". In Proceedings of CONCUR'01, volume 2154 of LNCS, pages 426-440, 2001.

12. P. Godefroid and R. Jagadeesan. "Automatic Abstraction Using Generalized ModelChecking”. In Proceedings of CAV'02, volume 2404 of LNCS, pages 137-150, 2002.

13. S. Graf and H. Saïdi. "Construction of Abstract State Graphs with PVS". In Proceedings of CAV'97, volume 1254 of $L N C S$, pages 72-83, 1997.

14. O. Grumberg, M. Lange, M. Leucker, and S. Shoham. "Don't Know in the $\mu$-Calculus". In Proceedings of CAV'05, volume 3385 of LNCS, pages 233-249, 2005.

15. A. Gurfinkel and M. Chechik. "Generating Counterexamples for Multi-Valued ModelChecking”. In Proceedings of FME'03, volume 2805 of LNCS, 2003.

16. A. Gurfinkel and M. Chechik. "Multi-Valued Model-Checking via Classical ModelChecking”. In Proceedings of CONCUR'03, volume 2761 of LNCS, pages 263-277, 2003.

17. A. Gurfinkel and M. Chechik. "How Thorough is Thorough Enough". In Proceedings of CHARME'05, volume 3725 of LNCS, pages 65-80, 2005.

18. A. Gurfinkel, O. Wei, and M. Chechik. "Systematic Construction of Abstractions for ModelChecking”. In Proceedings of VMCAI'06, volume 3855 of LNCS, pages 381-397, 2006.

19. S. Hazelhurst and C. H. Seger. "Model Checking Lattices: Using and Reasoning about Information Orders for Abstraction". Logic Journal of the IGPL, 7(3):375-411, May 1999.

20. T. Henzinger, R. Jhala, R. Majumdar, and G. Sutre. "Lazy Abstraction". In Proceedings of POPL'02, pages 58-70, 2002.

21. T. A. Henzinger, R. Jhala, R. Majumdar, and K. L. McMillan. "Abstractions from Proofs". In Proceedings of POPL'04, pages 232-244, 2004.

22. S. C. Kleene. Introduction to Metamathematics. New York: Van Nostrand, 1952.

23. D Kozen. "Results on the Propositional $\mu$-calculus". Theoretical Computer Science, 27 : 334-354, 1983.

24. K.G. Larsen and L. Xinxin. "Equation Solving Using Modal Transition Systems". In Proceedings of LICS'90, 1990.

25. C. Pasareanu, M. Dwyer, and W. Visser. "Finding Feasible Counter-examples when Model Checking Abstracted Java Programs". In Proceedings of TACAS'03, volume 2031 of LNCS, pages 284-298, 2003.

26. T.W. Reps, M. Sagiv, and R. Wilhelm. "Static Program Analysis via 3-Valued Logic". In Proceedings of CAV'04, volume 3114 of LNCS, pages 15-30, 2004.

27. S. Shoham and O. Grumberg. "Monotonic Abstraction-Refinement for CTL". In Proceedings of TACAS'04, volume 2988 of LNCS, 2004.

28. F. Somenzi. "CUDD: CU Decision Diagram Package Release”, 2001. 